\title{
Synergistic Anti-tumor Effect of KLF4 and Curcumin in Human Gastric Carcinoma Cell Line
}

\author{
Jun Ji, He-Shuang Wang, Yan-Yan Gao, Li-Min Sang, Li Zhang*
}

\begin{abstract}
Krüppel-like factor 4 is a transcription factor which plays an important role in development and progression of various carcinomas. Curcumin characterized by excellent anti-cancer properties is regarded as a serviceable natural compound used in carcinoma therapy. This study aimed at exploring the impact of KLF4 overexpression in cooperation with curcumin on the proliferation, apoptosis and invasion of human gastric carcinoma BGC823 cells. Flow cytometry analysis, CCK-8 assays, transwell assays and Western blot results showed that KLF4 overexpression combined with curcumin had significant anti-proliferation, pro-apoptosis and anti-invasion effects on BGC-823 cells. We also found that KLF4 had synergistic effects with curcumin, better promoting apoptosis and inhibiting proliferation and invasion of gastric carcinona cells. These results indicate that KLF4 could be used as a potential therapeutic target; curcumin could act as an auxiliary and provide a promising therapeutic strategy in stomach cancer.
\end{abstract}

Keywords: KLF4 - curcumin - stomach cancer - proliferation - migration - invasion

Asian Pac J Cancer Prev, 15 (18), 7747-7752

\section{Introduction}

Gastric carcinoma is one of the most invasive and aggressive malignancy, with serious threatens to patients' health. Gastric cancer is considered as the fourth most common cancer worldwide which is caused by eating disorders, Helicobacter pylori (HP), carcinogenic chemicals and so on (Ekinci et al., 2014; Gryko et al., 2014; Huang et al., 2014). It is investigated that dietary improvements and prevention in HP infection due to the use of antibiotics can effectively cause a steady decrease in incidence and mortality rates of stomach cancer (Ekinci et al., 2014). While the present situation of gastric cancer therapy is still barely satisfactory, it is imperative to get a detailed understanding of the molecular biology of gastric carcinoma. It will be undoubtedly a general trend that molecular therapy combined with anti-tumor natural compound are employed in gastric carcinoma treatment.

The Krüppel-like factor (KLFs) family transcription factors play important role in the regulation of multiple biological functions, including proliferation, apoptosis, differentiation, inflammation, migration and tumor formation (Tiwari et al., 2013). KLF4 known as a zinc finger type of transcription factor has been investigated highly expressed in the skin, intestine, testis, lung, bone and various tumor tissues. KLF4 also called gut-enriched Krllppel-like factor (GKLF) has drawn much attention due to its tumor suppressive activity and growth arrest. It is reported that KLF4 is downregulated in a number of cancers (Flandez et al., 2008, Zhang et al., 2012).
Yoon et al. (2005) found that KLF4 was sufficient in cell proliferation of colon cancer. Wei et al. (2005) reported that alteration of KLF4 expression plays a critical role in gastric cancer cell growth and apoptosis. Zhang et al. (2012) demonstrated that KLF4 inhibited the proliferation, invasion and metastasis of gastric cancer. However, its role in cancer is not fully conclusive as it is also identified to act as an oncogene in some cancers.

Curcumin, a polyphenolic natural product, is the main active secondary metabolite of tumeric extracted from the rhizomes of Curcuma longa, which is used widely in cooking and traditional Chinese medicines (Mehta et al., 2014; Zang et al., 2014). It is reported that curcumin has been employed as an anticancer compound which effectively inhibits proliferation, invasion, migration, angiogenesis and metastasis in various cancer cells involving colon cancer, breast cancer, lung cancer, thyroid cancer and prostate cancer (Feng et al., 2014; Xu et al., 2014). Increasing evidence indicates that curcumin has a range of molecular targets and influences numerous biochemical and molecular process. Moreover, curcumin is safe for humans and there is no dose-limiting toxicity when curcumin is administered at doses up to $10 \mathrm{~g} / \mathrm{day}$ (Lu et al., 2014; Xue et al., 2014).

In the present study, we attempt to estimate the effect of KLF4 overexpression cooperated with curcumin on proliferation, apoptosis and invasion of human gastric carcinoma cell line BGC-823 in vitro. In addition, we also identified whether curcumin had synergistic action with KLF4 for the purpose of providing insights toward gastric cancer treatment. 


\section{Materials and Methods}

\section{Cell culture, transfection and treatment}

The human gastric carcinoma BGC-823 cells were purchased from the American Type Culture Collection (Manassas, VA, USA) and cultured in RPMI medium (Gibco Invitrogen Corporation, Carlsbad, CA) supplemented with $10 \%$ Fetal bovine serum (Gibco Invitrogen Corporation, Carlsbad,CA), $2 \mathrm{mM} \mathrm{L-glutamine,}$ $100 \mathrm{IU} / \mathrm{ml}$ penicillin and $100 \mathrm{mg} / \mathrm{ml}$ streptomycin at $37^{\circ} \mathrm{C}$ in a 5\% $\mathrm{CO}_{2}$ incubator. Cells of KLF4 low-expression were screened for transfection by means of western blot. The plasmid pCMV-KLF4; provided by JRDUN Biotechnogy (Shanghai) co.Ltd. Plasmid KLF4 (3 mg) or mock-vehicle plasmid were transfected into BGC-823 cell lines in 6-well plates $(1 \mathrm{mg} / \mathrm{ml})$ using the lentiviral vector, according to the manufacturer's instructions. Cells were treated with curcumin $(15 \mu \mathrm{mol} / \mathrm{l})$ for $48 \mathrm{~h}$, and then were subjected to cell apoptosis and viability assay.

\section{Quantitative reverse transcription ( $q R T)-P C R$}

Total RNA was isolated from transfected cells, mock cells and non-transfected cells using TRIzol (Invitrogen) according to the protocol. High Capacity cDNA Reverse Transcription Kit (Applied Bios stems, Foster City, CA, USA) was employed to synthesize cDNAs. Quantitative real-time PCR was preceded using SYBR Green PCR Master Mix (Applied Bios stems) in a 7900 RealTime PCR System (Applied Bios stems). GADPH was performed as the reference gene. The following primers were used: for KLF4, 5' AACCTGGCGGACATCAAC 3' (forward), 5' AGCACGAACTTGCCCATC 3' (reverse); for GAPDH, 5' CACCCACTCCTCCACCTTTG 3' (forward), 5' CCACCACCCTGTTGCTGTAG 3' (reverse). The PCR cycles were $95^{\circ} \mathrm{C}$ for 10 minutes, followed by 40 cycles of $95^{\circ} \mathrm{C}$ for 15 seconds and $60^{\circ} \mathrm{C}$ for 1 minute. Each reaction was performed in triplicate and analyzed individually. The results were analyzed by ABI Prism 7300 SDS Software.

\section{Cell viability assay}

For cell viability assay, cells $\left(5 \times 10^{5}\right.$ cells $\left./ \mathrm{ml}\right)$ were loaded in 96-well plates and treated with DMSO for the indicated times. CCK8 $(10 \mu \mathrm{L})$ was added to each well with treated cells and incubated at $37^{\circ} \mathrm{C}$ for another $4 \mathrm{~h}$. Absorbance was read at $450 \mathrm{~nm}$ using a microplate reader.

\section{Cell apoptosis assay}

With or without curcumin treatment for 24 hours, cells were stained with annexin $\mathrm{V}$-fluorescein isothiocyanate and apoptosis rates were analyzed using a flow cytometer (FACSCalibur, BD Biosciences).

\section{Invasion assay}

The invasion assay was carried out by using a modified two chamber plates with a pore size of $8 \mu \mathrm{m}$. Cells were harvested and resuspended in serum free medium in the upper chamber coated with $150 \mathrm{mg}$ Matrigel (BD Biosciences, Bedford, MD). After $24 \mathrm{~h}$ incubation at $37^{\circ} \mathrm{C}$, no invasive cells were gently removed from the top of the matrigel with a cotton-tipped swab. Invasive cells at the bottom of the matrigel were fixed in methanol, stained with $1 \%$ crystal violet and counted under a microscope. Results were averaged from three independent experiments.

\section{Western blotting}

Proteins from cell simples were separated by SDS polyacrylamide gel electrophoresis, followed by electrotransfer to a nitrocellulose membrane by means of a transfer cell (Bio-Rad, Hercules, CA, USA). The proteins were then transferred to nitrocellulose membrane and incubated overnight at $4^{\circ} \mathrm{C}$ with the following antibodies: anti-cyclin D1, anti-E-cadherin, anti-Bax, anti-VEGF (Santa Cruze, CA, USA), anti-PI3K, anti-JNK, anti-ERK (Cell Signaling Technology, Beverly, MA, USA), antiBCL-2. Immunoreactive bands were detected by reaction with the ECL detection system reagents (Amersham, Arlington Heights, IL, USA) and exposure to X-ray film, which was the developed and photographed.

\section{Statistical analysis}

Results were expressed as mean values \pm SEM of three independent experiments. For statistical tests, Prism 5.0 (GraphPad Software, SanDiego, CA, USA) was used. $p$ values less than 0.05 were considered significant.

\section{Results}

KLF4 Overexpression and curcumin inhibit the proliferation of BGC-823 cell promote apoptosis

To identify the roles of KLF4 expression in BGC-823 cells proliferation and apoptosis by real time quantitative PCR, the mammalian cell expression plasmids for KLF4 overexpression were constructed. As shown in Figure 1A, compared with the mock, the level of KLF4 mRNA was increased strongly in BGC-823 cells transfected with the KLF4 expression plasmid. The western blotting Figure 1B intuitively showed the high KLF4 expression of pCMV-KLF4.

We further evaluate the effects of KLF4 overexpression and cooperation with curcumin on the proliferation of BGC-823 cell. We detected the OD $(450 \mathrm{~nm})$ value of BGC-823 cell by CCK-8 to generate cell growth curves. As shown in Figure 1C, mock treated with curcumin, KLF4 overexpression and KLF4 overexpression treated with curcumin significantly inhibits BGC-823 proliferation at $72 \mathrm{~h}$, as compared to the control group. Figure $1 \mathrm{D}$ and 1E) showed that the cells of mock treated with curcumin, pCMV-KLF4 and pCMV-KLF4 treated with curcumin significantly decreased in S and G2 compared with the mock. Flow cytometric analysis displayed that $\mathrm{G} 1$ arrest before the apoptosis appeared in BGC-823 cell.

KLF4 and curcumin surpress BGC-823 cell proliferation by regulating PI3K/Akt and JNK/MAPK signal pathways

$\mathrm{PI} 3 \mathrm{~K}$ and cyclinD1 are crucial proteins in PI3K/ Akt signal pathway regulating cell proliferation and cell cycle (Rusyn et al., 2000; Wang et al., 2001; Kim et al., 2009). JNK known as a stress-activated protein kinase involved in JNK/MAPK signal pathway play important role in regulating cell proliferation (Wang et al., 2001). As is shown in Figure 1F, the levels of $p$-PI3K, cyclinD1 
$\mathbf{A}$
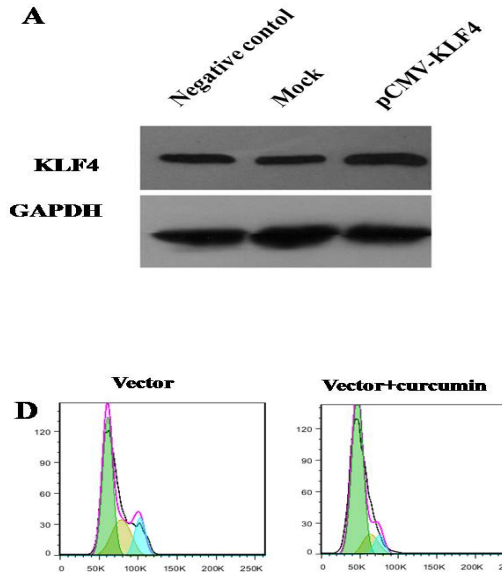

PCMV-KI.F4

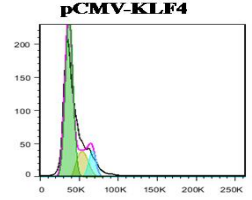

G

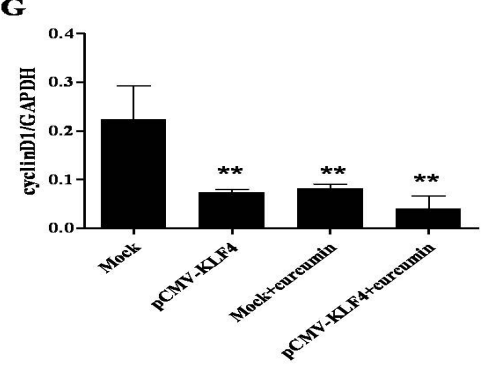

$\mathbf{B}$

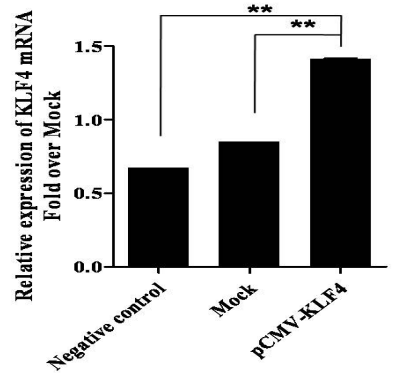

$\mathbf{E}$

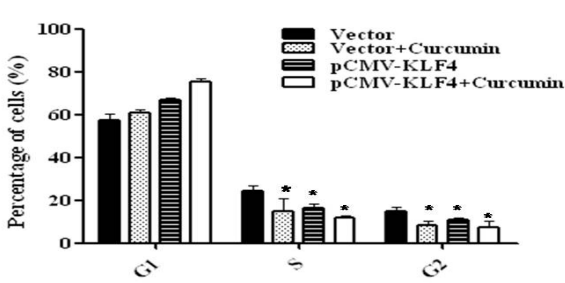

C

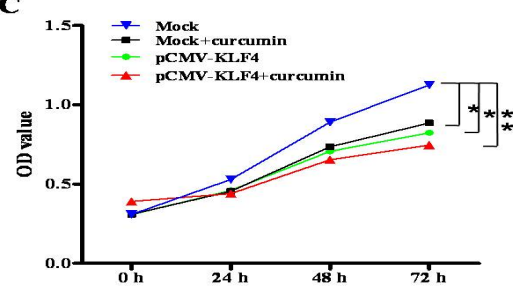

F

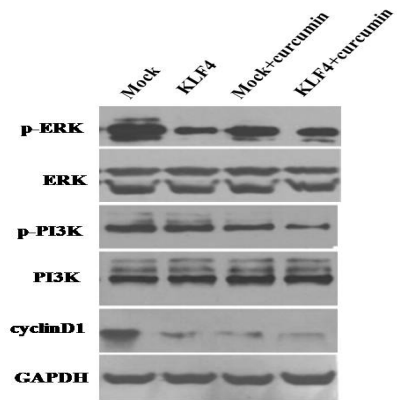

Figure 1. Effects of KLF4 Expression and Curcumin on Proliferation of BGC-823 Cells. A) BGC-823 cells were transfected with KLF4 plasmids. At $48 \mathrm{hr}$ post-transfection, cells were lysed for WB analysis at $24 \mathrm{hr}$ post-transfection using antibodies against the indicated proteins. GAPDH was detected as control for sample loading; B) Total RNA was isolated from cell samples and the mRNA level of KLF4 was analyzed by quantitative RT-PCR; C) Cell proliferation was measured at the indicated time post-transfection by CCK-8 test as described in the Methods and Materials; D, E) Effects of KLF4 expression and curcumin on cell-cycle distribution were detected by flow cytometry; F) Cells were transfected with the indicated plasmids or treated with curcumin and lysed for WB analysis at $24 \mathrm{~h}$ using antibodies against the indicated proteins. GAPDH was detected as control for sample loading; G, H, I) The cylinD1, $p$-PI3K and $p$-ERK contents of mock cells, curcumin treated cells, KLF4 overexpression cells and curcumin treated transfected cells. Data were presented as mean $\pm \mathrm{SD}, \mathrm{n}=3, * P<0.05, * * P<0.01, \Delta P<0.05, \triangle \triangle P<0.01$

and $p$-JNK are decreased in KLF4 expression plasmid transfected cells and curcumin treated transfected cells. In addition, the Figure $1 \mathrm{H}$ and $1 \mathrm{I}$ shows that $p$-PI3K and $p$-JNK levels of curcumin treated transfected cells are notable less than that of KLF4 overexpression cells. The results implied that KLF4 overexpression and cooperation with curcumin inhibit BGC-823 cell proliferation by regulating PI3K/Akt and JNK/MAPK signal pathways.

\section{KLF4 Overexpression and curcumin encourage the apoptosis of BGC-823 cell}

To estimate the effect of KLF4 overexpression, curcumin and combined action of both contribute to the inhibition of BGC-823 cell apoptosis, we examine the influence of them on cell apoptosis with Annexin-PI staining by flow cytometry. Figure $2 \mathrm{~A}$ and $2 \mathrm{~B}$ shows that mock treated with curcumin, KLF4 overexpression and KLF4 overexpression seeded with curcumin notably induce the apoptosis of human BGC-823 cells compared with the vector. In the Figure $2 \mathrm{~B}$, compared with the pCMV-KLF4, the pCMV-KLF4 treated with curcumin significantly promotes the apoptosis of BGC-823.
KLF4 and curcumin promote BGC-823 cell apoptosis by regulating apoptotic factors and ERK/MAPK signaling

As previous result showed that KLF4 overexpression and curcumin significantly accelerate the process of BGC823 cell apoptosis, we further measure the expression quality of apoptotic factors in mock cells, curcumin treated mock cells, pCMV-KLF4 plasmid-transfected cells and curcumin treated transfected cells (Figure 2C). The activation of Bax and Bcl-2 was detected by means of western blotting. Bax playing a positive role on apoptosis was significantly up-regulated compare to mock cells. On the contrary, the Bcl-2 expression in curcumin treated cells, and transfected cells seeded with curcumin are lower than the mock cells. The relative value of $\mathrm{Bax} / \mathrm{Bcl}-2$ was shown in Figure 2D. The Bax/Bcl-2 value of transfected cells treated with curcumin is notably higher than the transfected cells. Since ERK/MAPK signal pathway regulates the process of cell apoposis (McCain, 2013), we furthermore detected the expression level of $p$-ERK. The level of $p$-ERK was decreased notably in curcumin treated transfected cells compared with KLF4 overexpression cells (Figure 2F). These indicated that KLF4 and curcumin 

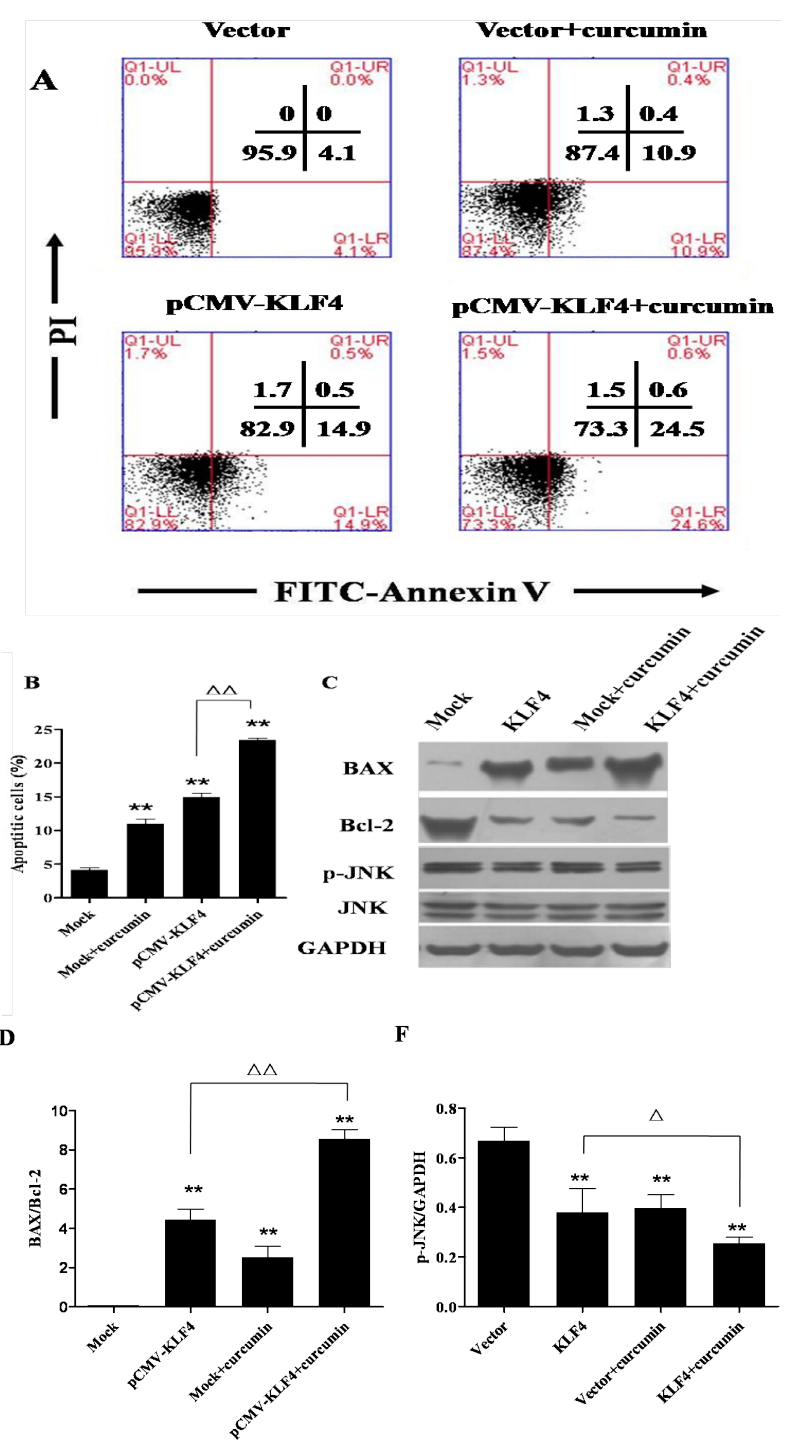

Figure 2. Effects of KLF4 Expression and Curcumin on Apoptosis of BGC-823 Cells. A, B) BGC-823 cells were transfected with KLF4 overexpression plasmids, control plasmids, or both treated with curcumin. After $48 \mathrm{hr}$, cell apoptosis was analyzed with Annexin-PI staining by flow cytometry; C, D, E) Cells were lysed for WB analysis at $24 \mathrm{~h}$ using antibodies against the indicated proteins including BAX, $\mathrm{Bcl}-2, p-\mathrm{JNK}$ and $p$-JNK. GAPDH was detected as control for sample loading. Data were presented as mean $\pm \mathrm{SD}, \mathrm{n}=3,{ }^{*} P<0.05$, $* * P<0.01, \triangle P<0.05, \triangle \triangle P<0.01$

promoted BGC-823 cell apoptosis by regulating apoptotic factors and ERK/MAPK signal pathway

KLF4 and curcumin inhibit invasion of BGC-823 cells by down-regulating E-cadherin level

The ability of cells to cross matrigel revealed the invasiveness of the BGC-823 cell. The effects of KLF4 and curcumin on invasion of human gastric carcinoma BGC-823 cells were identified by transwell assay. Figure $3 \mathrm{~A}$ implies that the vector treated with curcumin, pCMV-KLF4 and pCMV-KLF4 treated with curcumin significantly suppress the invasion of BGC-823 cell. Compared with the pCMV-KLF4, the effect of pCMVKLF4 treated with curcumin on BGC-823 cell invasion was more dramatic. The amount of invading cells crossing

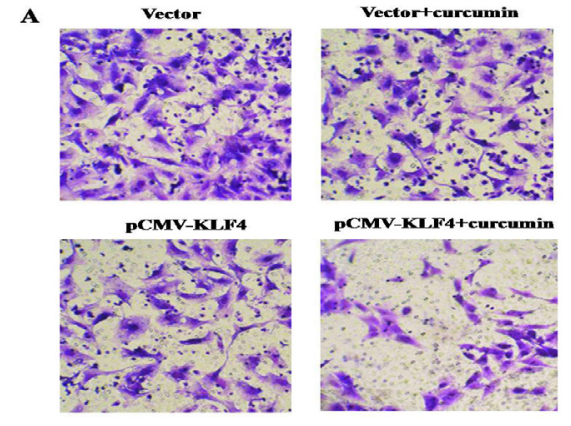

B

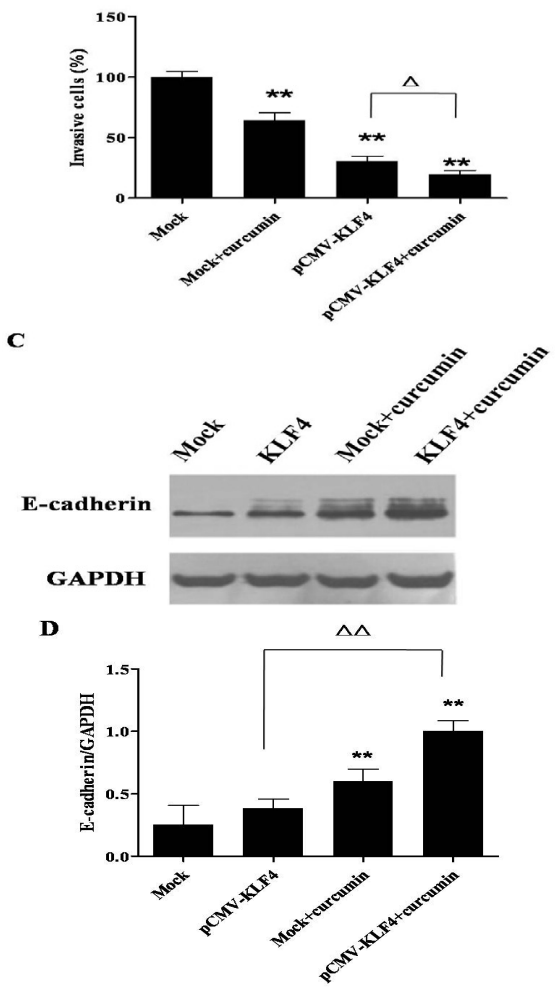

Figure 3. KLF4 Overexpression and Curcumin Inhibits Invasion of BGC-823 Cells. A, B) BGC-823 cell invasion assay was performed after re-expression of KLF4 BGC-823 cells, control group or KLF4 BGC-823 cells treated with curcumin; C, D) Cells were lysed for WB analysis at $24 \mathrm{~h}$ using antibodies against the indicated protein of E-cadherin. GAPDH was detected as control for sample loading. Data were presented as mean $\pm \mathrm{SD}, \mathrm{n}=3,{ }^{*} P<0.05,{ }^{*} * P<0.01,{ }^{\circ} P<0.05,{ }^{\triangle} \triangle P<0.01$

through the matrigel basement membrane is shown in Figure 3B.

E-cadherin is reported as a kind of $\mathrm{Ca}^{2+}$ dependent glycoprotein, which performs essential part in cell invasion and migration (Lubkov and Bar-Sagi, 2014; Pamies, 2014). As showed in Figure 3A and 3B, the levels of E-cadherin expression are higher in curcumin treated cells, KLF4 plasmid transfected cells and curcumin treated transfected cells. In the Figure 3C and 3D, the levels of E-cadherin are notably higher in curcumin treated cells, KLF4 overexpression cells and both treated cell compared with the control group. The E-cadherin level of curcumin treated transfected cells is significantly higher than that of KLF4 expression plasmid transfected cells. The results implied that KLF4 and curcumin inhibit invasion of BGC823 cells by down-regulating E-cadherin level. 


\section{Discussion}

Stomach cancer is the second-greatest cause of cancer death globally and patients died from gastric carcinoma are more than those died from other malignancies in China. Both molecular therapy and natural anti-cancer product treatment toward stomach cancer are immediate areas of research focus. KLF4, a transcription factor expressed abundantly in the epithelium of the digestive tract, has been reported that KLF4 expression plays a critical role in gastric cancer development and progression (Wei et al., 2005). Curcumin as a common natural food pigment exhibit favorable anti-cancer activity which be used in many kinds of neoplasms treatment. Our study including identifying the anti-proliferation, pro-apoptotic and anti-invasive effect and mechanism of KLF4, curcumin and their combination may help to better understand how KLF4 and curcumin affect gastric carcinoma cell physiological activity and provide theoretical basis for clinical early diagnosis and intervention of gastric cancer.

The dominate findings of this study are the following: i) KLF4, curcumin and their combination can effectively suppress the proliferation of human gastric carcinoma BGC-823 cells. The anti-proliferation effect of KLF4 overexpression and curcumin combination was more significantly than either of them. The impact of KLF4 and curcumin on cell circle result showed that KLF4, curcumin and their combination arrested cell cycle at G1 period. PI3K/Akt and JNK/MAPK single pathways have been reported as import pathways that closely correlate with the proliferation, differentiation and migration of tumor cells (Hinoi et al., 2014; Li et al., 2014). Western blotting result implied that the levels of $p$-PI3K, cyclinD1 and $p$-ERK in KLF4, curcumin and their combination were plummeting compared with the control, and the combination of KLF4 overexpression and curcumin lead notable decrease of $p$-PI3K, cyclinD1 and $p$-ERK. These findings indicated that KLF4 and curcumin can effectively inhibit the proliferation of human gastric cancer BGC-823 cells by regulating PI3K/Akt and ERK/MAPK single pathways and arrested cell cycle at G1 period by downregulating the expression of cyclinD1 (Meng et al., 2012). The combination of KLF4 overexpression and curcumin play a better role than either of them individually. ii) KLF4, curcumin and their co-work play a positive part in BGC-823 cell apoptosis. The cooperation of KLF4 overexpression and curcumin exhibited a more significant efficiency in inducing the process of apoptosis according to the flow cytometry analysis. Bax and Bcl-2 are the proteins which play important role in cell apoptosis (Theron et al., 2013; Pryczynicz et al., 2014). The further study on evaluating apoptotic proteins levels of BAX and Bcl-2 arrived at the consistent conclusion. JNK/MAPK signal pathway play crucial roles in tumor cell apoptosis (Meng et al., 2012; You et al., 2013; Bloch et al., 2014). p-JNK levels of KLF4 overexpression cells, curcumin treated cells and co-treated cells were notable less than the control. The above results explained that KLF4 promoted the apoptosis of BGC-823 cells by regulating JNK-MAPK signal pathway and combined with curcumin can achieve better result.
Previous study reported that KLF4 expression plays a critical role in gastric cancer development and progression (Wei et al., 2005; Zhang et al., 2012). Effect of KLF4 on gastric carcinoma BGC-823 cell invasion is discussed in our study. The result of whether KLF4 suppresses the invasion of BGC-823 cell showed that overexpression of KLF4 and combined with curcumin can effectively depress the invasion of gastric carcinoma cells. The western blotting result showed the expression level of E-cadherin was decreased more significantly in curcumin treated KLF4 overexprssion cells.

In conclusion, this study identified the anti-apoptotic/ pro-proliferative and anti-invasive roles of KLF4 and cowork with curcumin in human gastric carcinoma BGC823 cells, and explained the corresponding mechanism. These findings may provide the insights toward the molecular mechanism of stomach cancer progression and pathogenesis and benefit the development of therapeutical approaches for the disease.

\section{References}

Bloch O, Amit-vazina M, Yona E, et al (2014). Increased ERK and JNK activation and decreased ERK/JNK ratio are associated with long-term organ damage in patients with systemic lupus erythematosus. Rheumatology, $\mathbf{5 3}(\mathbf{6})$, 1034-42.

Ekinci AS, Bal O, Ozatli T, et al (2014). Gastric carcinoma with bone marrow metastasis: a case series. J Gastric Cancer, 14, 54-7.

Feng J, Tao T, Yan W, Chen CS, Qin X (2014). Curcumin inhibits mitochondrial injury and apoptosis from the early stage in EAE mice. Oxid Med Cell Longev, 2014, 728751.

Flandez M, Guilmeau S, Blache P, Augenlicht LH (2008). KLF4 regulation in intestinal epithelial cell maturation. Experimental Cell Res, 314, 3712-23.

Gryko M, Guzinska-ustymowicz K, Kisl UK J, et al (2014). High Fas expression in gastric carcinoma cells as a factor correlating with the occurrence of metastases to regional lymph nodes. Adv Med Sci, 59, 47-51.

Hinoi E, Iezaki T, Fujita H, et al (2014). PI3K/Akt is involved in brown adipogenesis mediated by growth differentiation factor-5 in association with activation of the Smad pathway. Biochem Biophys Res Commun, 450, 255-60.

Huang G, Dang ZF, Dang YM, et al (2014). Expression and underlying roles of IGFBP-3 in paclitaxel-treated gastric cancer Sgc-7901 cells. Asian Pac J Cancer Prev, 15, 5741-5.

Kim SJ, Yoo KY, Jeong CW, et al (2009). Urinary trypsin inhibitors afford cardioprotective effects through activation of PI3K-Akt and ERK signal transduction and inhibition of p38 MAPK and JNK. Cardiology, 114, 264-70.

Li Z, Lu H, Li ZB (2014). The PI3K/Akt signalling pathway may play an internal role related to abnormal condylar growth: a preliminary study. Int J Oral Maxillofac Surg, [Epub ahead of print].

Lu Y, Wei C, Xi Z (2014). Curcumin suppresses proliferation and invasion in non-small cell lung cancer by modulation of MTA1-mediated Wnt/beta-catenin pathway. In Vitro Cell Dev Biol Anim, [Epub ahead of print].

Lubkov V, Bar-sagi D (2014). E-cadherin-mediated cell coupling is required for apoptotic cell extrusion. Curr Biol, 24, 868-74.

Mccain J (2013). The MAPK (ERK) pathway: investigational combinations for the treatment of BRAF-mutated metastatic melanoma. $P$ T, 38 (2), 96-108.

Mehta HJ, Patel V, Sadikot RT (2014). Curcumin and lung 
cancer-a review. Target Oncol, [Epub ahead of print].

Meng FM, Yang JB, Yang CH, et al (2012). Vitexicarpin induces apoptosis in human prostate carcinoma PC-3 cells through G2/M phase arrest. Asian Pac J Cancer Prev, 13, 6369-74.

Pamies P (2014). E-cadherin-guided migration. Nat Mater, 13, 664 .

Pryczynicz A, Gryko M, Niewiarowska K, et al (2014). Bax protein may influence the invasion of colorectal cancer. World J Gastroenterol, 20, 1305-10.

Rusyn EV, Reynolds ER, Shao H, et al (2000). Rit, a non-lipidmodified Ras-related protein, transforms NIH3T3 cells without activating the ERK, JNK, p38 MAPK or PI3K/Akt pathways. Oncogene, 19, 4685-94.

Theron KE, Penny CB, Hosie MJ (2013). The Bax/Bcl-2 apoptotic pathway is not responsible for the increase in apoptosis in the RU486-treated rat uterus during early pregnancy. Reprod Biol, 13, 290-7.

Tiwari N, Meyer-schaller N, Arnold P, et al (2013). Correction: Klf4 is a transcriptional regulator of genes critical for EMT, including Jnk1. PLoS One, 8 (2), e57329.

Wang Z, Jiang C, Ganther H, Lu J (2001). Antimitogenic and proapoptotic activities of methylseleninic acid in vascular endothelial cells and associated effects on PI3K-AKT,ERK, JNK and p38 MAPK signaling. Cancer Res, 61, 7171-8.

Wei D, Gong W, Kanai M, et al (2005). Drastic down-regulation of Kruppel-like factor 4 expression is critical in human gastric cancer development and progression. Cancer Res, 65, 2746-54.

Xu XB, Chen B, Liu WY (2014). Curcumin inhibits the invasion of thyroid cancer cells via down-regulation of PI3K/Akt signaling pathway. Gene, 546, 226-32.

Xue X, Yu JL, Sun DQ, et al (2014). Curcumin induces apoptosis in SGC-7901 gastric adenocarcinoma cells via regulation of mitochondrial signaling pathways. Asian Pac J Cancer Prev, 15, 3987-92.

Yoon HS, Ghaleb AM, Nandan MO, et al (2005). Kruppellike factor 4 prevents centrosome amplification following gamma-irradiation-induced DNA damage. Oncogene, 24, 4017-25.

You H, Lei P, Andreadis ST (2013). JNK is a novel regulator of intercellular adhesion. Tissue Barriers, 1, 268-45.

Zang S, Liu T, Shi J, Qiao L (2014). Curcumin: a promising agent targeting cancer stem cells. Anticancer Agents Med Chem, 14, 787-92.

Zhang N, Zhang J, Shual L, et al (2012). Kruppel-like factor 4 negatively regulates beta-catenin expression and inhibits the proliferation, invasion and metastasis of gastric cancer. Int J Oncol, 40, 2038-48. 